\title{
Mid-infrared intersubband absorption from p-Ge quantum wells grown on Si substrates
}

Cite as: Appl. Phys. Lett. 108, 091114 (2016); https://doi.org/10.1063/1.4943145

Submitted: 21 December 2015 . Accepted: 20 February 2016 . Published Online: 04 March 2016

K. Gallacher (D), A. Ballabio, R. W. Millar, J. Frigerio, A. Bashir, I. MacLaren (D), G. Isella (D), M. Ortolani (D), and D. J. Paul (D)

\section{ARTICLES YOU MAY BE INTERESTED IN}

Interfacial sharpness and intermixing in a Ge-SiGe multiple quantum well structure Journal of Applied Physics 123, 035703 (2018); https://doi.org/10.1063/1.5001158

8-band k.p modelling of mid-infrared intersubband absorption in Ge quantum wells Journal of Applied Physics 120, 043103 (2016); https://doi.org/10.1063/1.4959259

Germanium-on-silicon nitride waveguides for mid-infrared integrated photonics

Applied Physics Letters 109, 241101 (2016); https://doi.org/10.1063/1.4972183

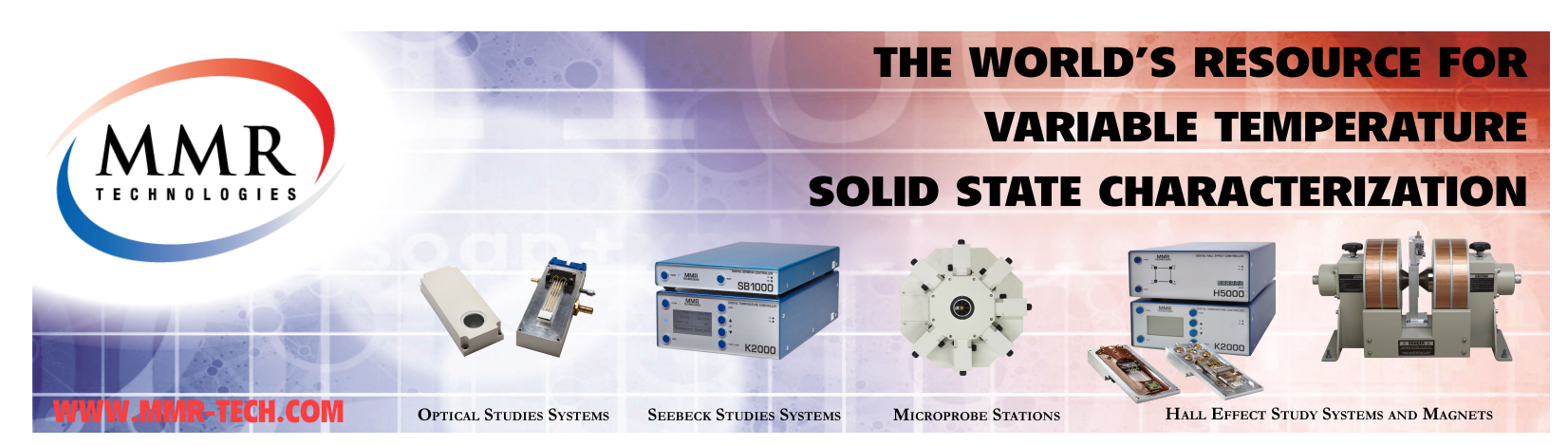
VARIABLE TEMPERATURE 


\title{
Mid-infrared intersubband absorption from p-Ge quantum wells grown on Si substrates
}

\author{
K. Gallacher, ${ }^{1}$ A. Ballabio, ${ }^{2}$ R. W. Millar, ${ }^{1}$ J. Frigerio, ${ }^{2}$ A. Bashir,${ }^{3}$ I. MacLaren, ${ }^{3}$ G. Isella, ${ }^{2}$ \\ M. Ortolani, ${ }^{4}$ and D. J. Paul ${ }^{1, a)}$ \\ ${ }^{1}$ School of Engineering, University of Glasgow, Rankine Building, Oakfield Avenue, Glasgow G12 8LT, \\ United Kingdom \\ ${ }^{2}$ L-NESS, Dipartimento di Fisica del Politecnico di Milano, Polo Territoriale di Como, Via Anzani 42, \\ Como I-22100, Italy \\ ${ }^{3}$ School of Physics and Astronomy, University of Glasgow, Kelvin Building, University Avenue, \\ Glasgow G12 8QQ, United Kingdom \\ ${ }^{4}$ Center for Life NanoScience@Sapienza, Istituto Italiano di Tecnologia, Viale Regina Elena 291, \\ Rome I-00161, Italy
}

(Received 21 December 2015; accepted 20 February 2016; published online 4 March 2016)

\begin{abstract}
Mid-infrared intersubband absorption from p-Ge quantum wells with $\mathrm{Si}_{0.5} \mathrm{Ge}_{0.5}$ barriers grown on a Si substrate is demonstrated from 6 to $9 \mu \mathrm{m}$ wavelength at room temperature and can be tuned by adjusting the quantum well thickness. Fourier transform infra-red transmission and photoluminescence measurements demonstrate clear absorption peaks corresponding to intersubband transitions among confined hole states. The work indicates an approach that will allow quantum well intersubband photodetectors to be realized on Si substrates in the important atmospheric transmission window of 8-13 $\mu \mathrm{m}$. (C) 2016 AIP Publishing LLC. [http://dx.doi.org/10.1063/1.4943145]
\end{abstract}

There is a significant interest to develop cheap and practical detectors that cover the important transmission windows within the mid-infrared (MIR) (3-5 and $8-13 \mu \mathrm{m}$ wavelength) to enable on-chip biological and gas sensing spectroscopic detectors. ${ }^{1,2}$ Currently, the gold standard for detection at these wavelengths is mercury-cadmium-telluride (MCT) due to the inherently large detectivities from interband absorption. ${ }^{3}$ The disadvantages with MCT are that it is fragile, has low process uniformity, is not easily integrated with $\mathrm{Si}$, and the toxic elements have been banned for use in civilian products in Europe. The InSb gas detectors are now available in the $3-5 \mu \mathrm{m}$ window, but the material is fragile and difficult to process. ${ }^{4}$

A good candidate for cheap and practical sensors is the quantum well (QW) infrared photodetectors (QWIPs). Since absorption can occur from intersubband transitions within the QW, the absorption can be tuned by adjusting the QW width, thereby changing the energy of the confined subband states. This allows the potential to engineer strong absorption from the normally transparent semiconductors like $\mathrm{Si}$ and Ge in the MIR. QWIPs have been previously demonstrated with $\mathrm{p}$ and n-type SiGe designs. ${ }^{5,6}$ There has been only one recent demonstration of intersubband absorption from a pure Ge QW (n-type). ${ }^{7}$ The majority of the investigation into Si compatible QWIPs has been limited to the low Ge concentration structures. This has been due to the difficulty in growing the high quality Ge rich superlattices on $\mathrm{Si}$, due to the large lattice mismatch of $4.2 \%$ between $\mathrm{Si}$ and Ge. ${ }^{8}$ Instead of using the $\geq 5 \mu \mathrm{m}$ thick graded buffers, it is now possible to grow the high quality pure Ge QWs on a thin $(\sim 1 \mu \mathrm{m})$ SiGe strain relaxed virtual substrate ${ }^{9,10}$ using techniques such as low energy plasma enhanced chemical vapor deposition (LEPECVD). The benefit of using p-Ge

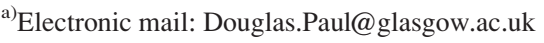

QWs over $\mathrm{p}-\mathrm{Si}_{1-x} \mathrm{Ge}_{x} \mathrm{QWs}$ for $x<0.85$ is that it will provide larger absorption coefficients, due to the smaller effective masses for both the heavy hole $(\mathrm{HH})$ and light hole (LH) bands. In addition, whilst conduction band designs allow only the TM (z)-polarized intersubband transitions, the valence band allows both TE (x-y) and TM polarizations to be observed. ${ }^{8}$ This arises due to non-parabolicity effects and therefore strong coupling of the bands, which relaxes parabolic selection rules. ${ }^{8,11,12}$ Hence, both surface normal and waveguide geometry devices can be realized. In this paper, surface-normal p-type Ge QW intersubband absorption is investigated for a range of QW widths designed by 8-band k.p theory ${ }^{13}$ and analyzed using Fourier transform infrared (FTIR) spectroscopy, X-ray diffraction (XRD), Raman spectroscopy, and scanning transmission electron microscopy (STEM).

The band energies and confined wave-functions were calculated using a self-consistent 8-band k.p PoissonSchrödinger solver with periodic boundary conditions orientated along the growth-axis and the deformation potentials from Ref. 13. Fig. 1 presents the calculated band structure for a $5.4 \mathrm{~nm} \mathrm{Ge} \mathrm{QW}$ and $3.6 \mathrm{~nm} \mathrm{Si}_{0.5} \mathrm{Ge}_{0.5}$ barriers with a residual tensile strain of $0.2 \%$ in the $\mathrm{Si}_{0.2} \mathrm{Ge}_{0.8}$ relaxed buffer. It is clear that the ground state in the QW is $\mathrm{HH} 1$ due to strain splitting of the $\mathrm{HH}$ and $\mathrm{LH}$ bands. The $\mathrm{HH} 2$ and $\mathrm{HH} 3$ are at roughly the same energy as the LH1 and LH2 subband states, respectively. For a doping level of $5 \times 10^{18} \mathrm{~cm}^{-3}$ within the $\mathrm{QW}$, the Fermi level at room temperature sits at the $\mathrm{HH} 2$ band. Therefore, the HH2-HH3 intersubband transition should be optically active. A larger doping concentration leads to a greater population of the $\mathrm{HH} 2$ band and should provide a stronger absorption coefficient. ${ }^{14}$

The designed Ge QW structures were grown by LEPECVD on high resistivity $\mathrm{Si}(100)$ to reduce free-carrier absorption from the substrate. A $500 \mathrm{~nm}$ thick $\mathrm{Si}_{0.6} \mathrm{Ge}_{0.4}$ 


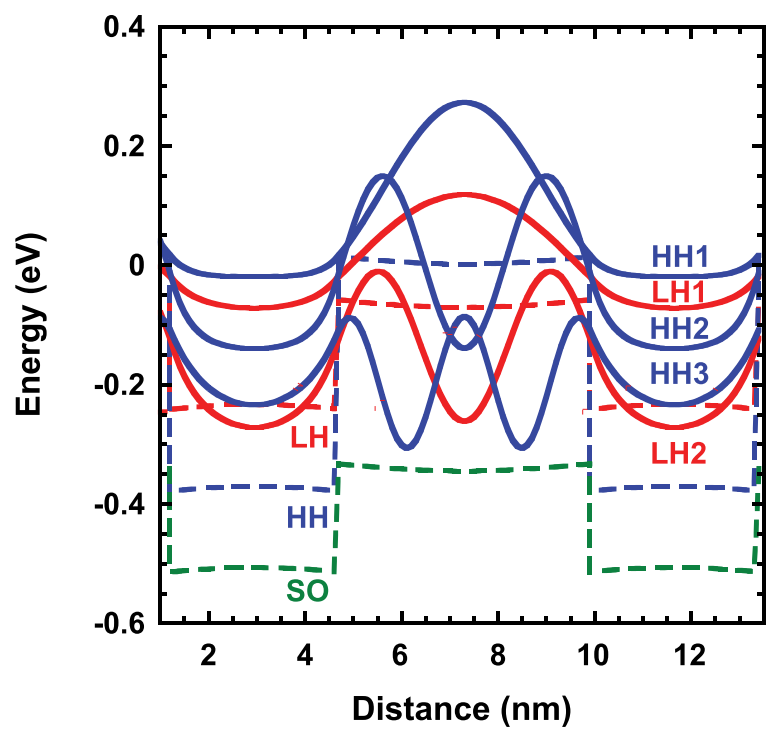

FIG. 1. A schematic diagram of the calculated band structure for a 5.4 wide Ge quantum well sandwiched between $3.6 \mathrm{~nm} \mathrm{Si}_{0.5} \mathrm{Ge}_{0.5}$ barriers. The squared wave-functions for the lowest energy subband states for the heavy hole $(\mathrm{HH})$ and light hole $(\mathrm{LH})$ bands are shown.

layer was first grown followed by a $500 \mathrm{~nm}$ linear graded buffer from $\mathrm{x}=0.4$ until $\mathrm{x}=0.8$. Then, an undoped $10 \mathrm{~nm}$ $\mathrm{Si}_{0.2} \mathrm{Ge}_{0.8}$ spacer region was grown followed by the evenly doped active region $\left(\mathrm{N}_{A} \approx 5 \times 10^{18} \mathrm{~cm}^{-3}\right)$ consisting of 500 periods of compressively strained Ge QWs (tensile strained $\mathrm{Si}_{0.5} \mathrm{Ge}_{0.5}$ barriers) of $5.4(3.6), 8.1(5.4)$, or $9.2(6.1) \mathrm{nm}$ thickness. Finally, another undoped $10 \mathrm{~nm} \mathrm{Si}_{0.2} \mathrm{Ge}_{0.8}$ spacer layer was grown, followed by a $20 \mathrm{~nm} \mathrm{Si}_{0.2} \mathrm{Ge}_{0.8}$ cap region. The total heterostructure thicknesses range from $\sim 4$ to $7 \mu \mathrm{m}$. The graded buffer layers and the fully strained state of the superlattice were observed by XRD. Fig. 2 shows the XRD spectra for the $8.1 \mathrm{~nm}$ QW structure. The $\mathrm{Si}(004)$ peak of the substrate is visible at $\mathrm{q}=7.365 \mathrm{~nm}^{-1}$ and is used as a reference. The superlattice fringes are visible with a spacing that indicates a period of $\sim 12 \mathrm{~nm}$. The zeroth-order fringe at $7.051 \mathrm{~nm}^{-1}$ is matched with the virtual substrate and

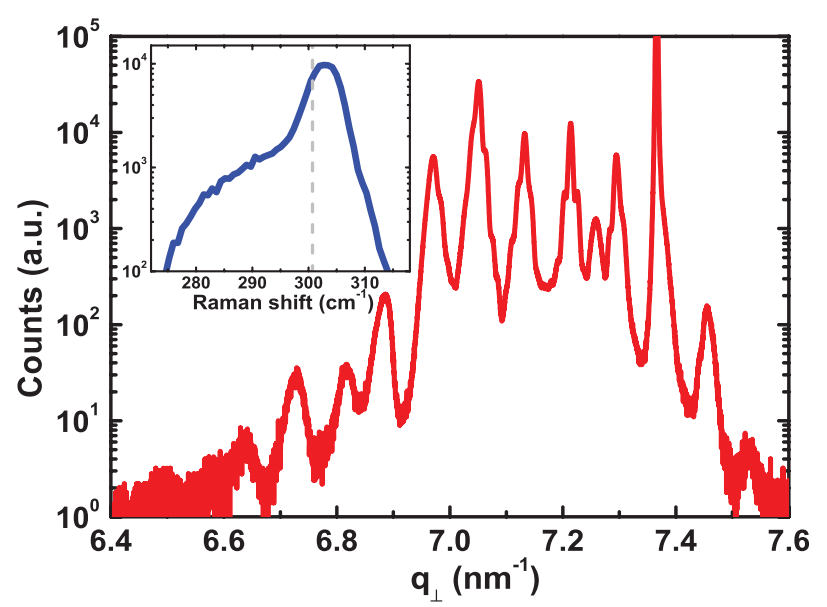

FIG. 2. X-ray diffraction plot (theta scan) of the $8.1 \mathrm{~nm}$ wide Ge quantum well $(\mathrm{QW})$ structure with $5.4 \mathrm{~nm} \mathrm{Si}_{0.5} \mathrm{Ge}_{0.5}$ barriers showing the narrow pattern of symmetrical Bragg peaks indicative of long-range periodicity of the heterostructure. The inset is the Raman spectrum showing the convolution of the Ge-Ge optical phonon line between the Ge QW and SiGe barrier. For reference, the bulk Ge phonon frequency is shown (grey dashed). indicates an average Ge content of $80 \%$ in the active layer stack. The high quality of the heterostructure is testified by the narrow peaks in the X-ray diffraction spectra (see Fig. 2).

Raman spectroscopy was directly performed on the $8.1 \mathrm{~nm}$ QW sample by dry etching the cap and spacer region. With a pump at $532 \mathrm{~nm}$ wavelength, this corresponds to a penetration depth of $\sim 20 \mathrm{~nm}$; therefore, it should probe a full period of the active region. There is a convolution of the Ge-Ge phonon peak for the Ge QW and $\mathrm{Si}_{0.5} \mathrm{Ge}_{0.5}$ barrier, and this is shown within the inset of Fig. 2. The Ge-Ge line of $\sim 302.7 \mathrm{~cm}^{-1}$ for the QW indicates a compressive strain of $\sim 0.39 \% .{ }^{15}$ The Ge-Ge, Si-Ge, and $\mathrm{Si}$-Si phonon peaks (not shown) indicate a tensile strain of $\sim 0.2 \%$ for the SiGe barrier. ${ }^{16}$ This demonstrates that the Ge rich superlattice structure has been properly strain symmetrized.

The heterolayer thicknesses were measured by STEM. The STEM was performed on a probe-corrected JEOL ARM 200F equipped with a cold field emission gun operated at $200 \mathrm{kV}$. A Gatan GIF Quantum ER energy filter/spectrometer equipped with a fast dual electron energy loss spectroscopy (EELS) system was used. The STEM was operated in the high angle annular dark field (HAADF) mode. The atomic (Z) contrast image was obtained by the detection of elastically and quasi-elastically scattered electrons using a HAADF. Therefore, this is a well suited method for imaging Ge and SiGe interfaces. The HADDF STEM image in Figs. 3(a) and 3(b) shows the extremely smooth and abrupt interfaces between the Ge QWs and the $\mathrm{Si}_{0.5} \mathrm{Ge}_{0.5}$ barriers. The false
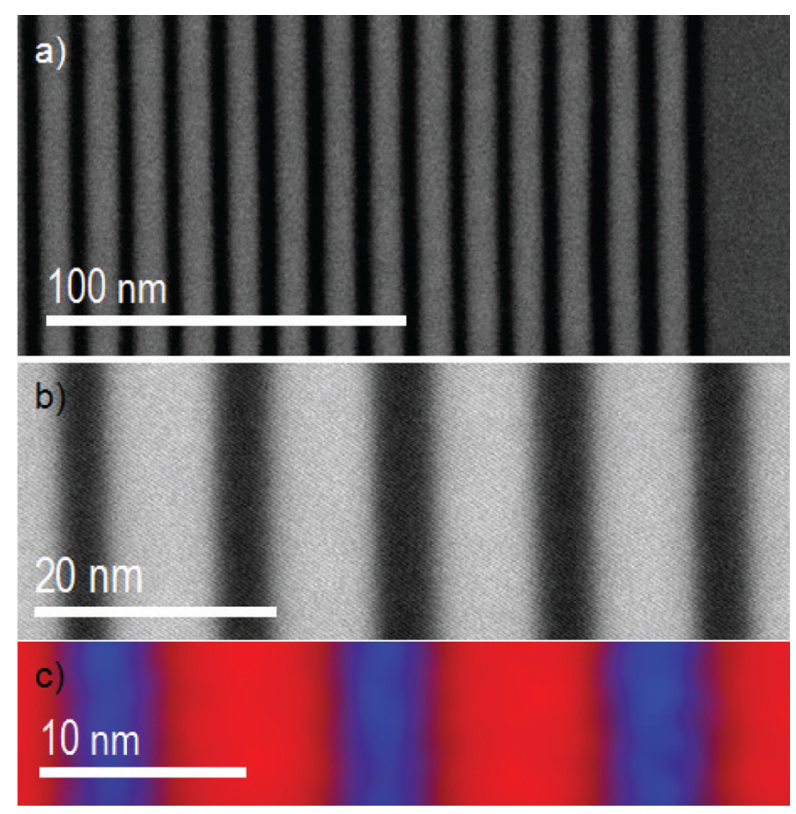

FIG. 3. (a) A cross-sectional scanning transmission electron microscope (STEM) image of the $8.1 \mathrm{~nm}$ Ge quantum well (QW) structure; it clearly distinguishes between the layered structure and the capping layer. (b) A (HAADF) STEM image at $3 \times 10^{6}$ times magnification. Due to the $\mathrm{Z}^{2}$ dependence, the heavier atoms appear brighter because of strong scattering at high angles while the lighter atoms appear darker. Therefore, the lighter regions in this image correspond to Ge QWs, and the darker regions correspond to the $\mathrm{SiGe}$ barriers. (c) An elemental map obtained from EELS spectrum imaging from a different region. Red is Ge, and blue is $\mathrm{Si}_{0.5} \mathrm{Ge}_{0.5}$. 
color HAADF STEM image in Fig. 3(c) reveals that there is minimal interdiffusion caused during the growth of the active layers, indicating that the potential barrier for holes is very steep. False color was added from an EELS analysis. Red in the image denotes Ge and blue is $\mathrm{Si}_{0.5} \mathrm{Ge}_{0.5}$.

The FTIR transmission measurements were performed on the as-grown QWs in vacuum at temperatures ranging from 6 to $300 \mathrm{~K}$. The setup consisted of a Bruker IFS66v interferometer and a nitrogen-cooled MCT detector. Blank chips from the wafers were bonded onto the cold finger of an optical cryostat aligned within the sample chamber of the FTIR. The measurements were performed in surface normal (x-y) geometry with in-plane (TE) light polarization state defined by the properties of the Michelson interferometer. The electric field component parallel to the growth axis (TM) was null at all wavelengths before hitting the sample, but due to the refractive index variation of the complete structure and the backside of the substrate being unpolished, there will be some scattering that couples some of the radiation into the TM polarized active transitions, so both the TE and TM transitions will be observed. ${ }^{11,17}$ The normalized FTIR absorption spectra at $300 \mathrm{~K}$ in vacuum for the three different QW widths are demonstrated in Fig. 4(a). The very narrow absorption peak at $\sim 9 \mu \mathrm{m}$ wavelength, which is visible in all the spectra, corresponds to
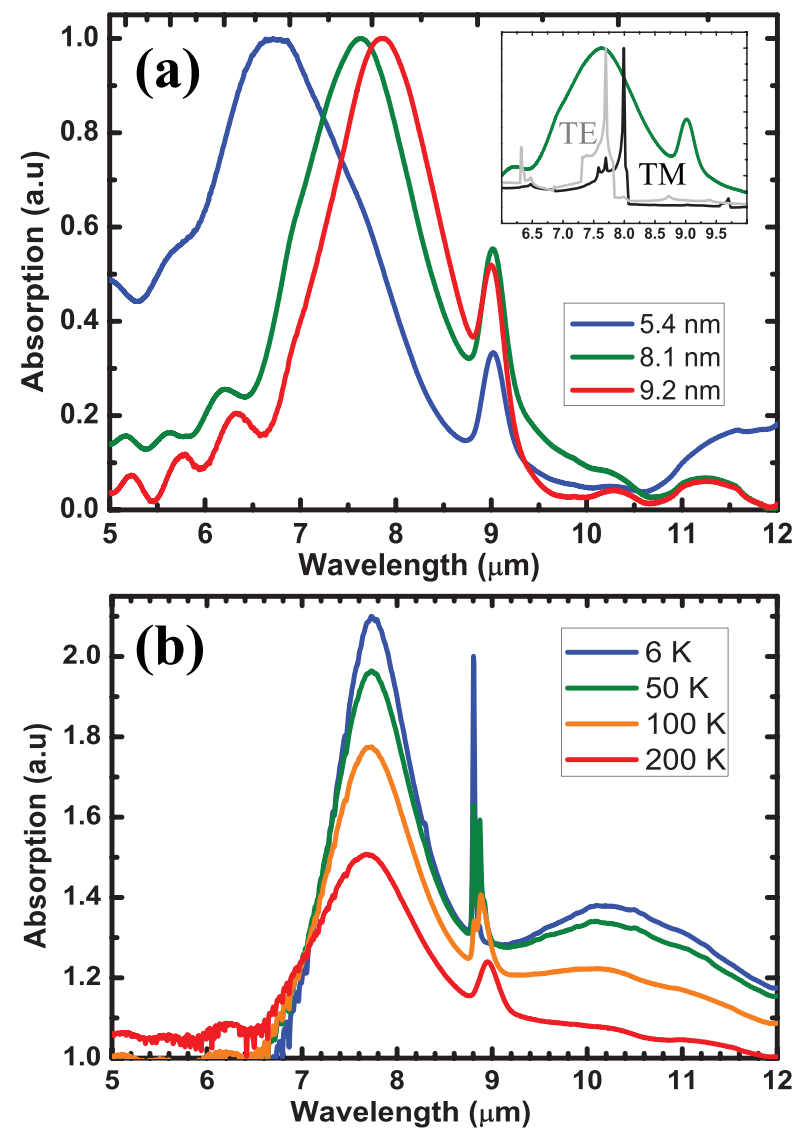

FIG. 4. (a) Fourier transform infra-red (FTIR) absorption spectra at $300 \mathrm{~K}$ under vacuum for the as-grown 5.4, 8.1, and 9.2 nm Ge quantum well (QW) structures. The inset shows the FTIR absorption spectra of the $8.1 \mathrm{~nm} \mathrm{QW}$ structure and the modelled intersubband absorption spectra for TM and TE transitions. (b) Low temperature FTIR absorption spectra of the $8.1 \mathrm{~nm}$ QW structure from 6 to $200 \mathrm{~K}$. vibrational interstitial oxygen impurities in the $\mathrm{Si}$ substrate. $^{18}$

The QW design was for a bound-to-continuum transition from the $\mathrm{HH} 2$ to the mixed $\mathrm{LH}$ continuum formed from weakly/unconfined $\mathrm{LH} 2$ state that mixes with the $\mathrm{HH} 3$ bound state. The inset in Fig. 4(a) shows the experimental absorption for the $8.1 \mathrm{~nm}$ QW design and the modelled calculation for an ideal TM and TE polarized light beam. The TM absorption corresponds to a $\mathrm{HH} 2-\mathrm{HH} 3$ transition, and the TE absorption corresponds to a LH1-HH3 transition. The model agrees reasonably well with the experimental peak positions but at present underestimates the absorption width for all of the designs. This is due the modelling not being able to account for broadening effects such as inhomogenous broadening. In Fig. 4(a), there is a clear shift in intersubband absorption to longer wavelengths for thicker QWs, which is expected, due to the decrease in intersubband energy level spacing with the increasing QW width. This illustrates how the absorption can be tuned by changing the QW widths. The low temperature $(6-200 \mathrm{~K})$ absorption spectra of the $8.1 \mathrm{~nm}$ QW structure are demonstrated in Fig. 4(b). In this instance, the absorption peak from the Si-O interstitial defect can be used as a temperature reference since it blue shifts with decreasing temperature and begins to exhibit a narrow line-width below $10 \mathrm{~K},{ }^{18}$ which is evident in the $6 \mathrm{~K}$ spectra. It is clear from Fig. 4(b) that as the temperature decreases the intersubband absorption is increasing. There is a negligible absorption shift observed with the decreasing temperature. This arises from how both the band-edges of the barriers and QWs are changing at approximately the same rate in energy and produce a negligible change in terms of the QW depth for both the HH and LH bands. ${ }^{19,20}$ It is also evident from the spectra that there is a longer wavelength absorption peak appearing with the decreasing temperature at $\sim 10.2 \mu \mathrm{m}$ wavelength. Most likely, the Fermi level enters the HH1 subband at low temperature, activating the HH1$\mathrm{HH} 2$ transition. The doping-dependent analysis will be needed to address this point further.

To further probe the intersubband transitions of the Ge QWs, the photoluminescence (PL) measurements were performed at $6 \mathrm{~K}$. The QWs were optically pumped using a continuous-wave solid-state diode laser emitting at $632 \mathrm{~nm}$ with an output power of $300 \mathrm{~mW}$. This corresponds to interband pumping of holes high in energy $(\sim 1.96 \mathrm{eV})$ above the Ge QW. PL occurs from these holes radiatively transitioning between the confined subbands. The PL measurements were operated in step-scan mode, using a $1 \mathrm{kHz}$ optical chopper and a lock-in amplifier to discriminate against ambient blackbody. This is especially important in the MIR as ambient blackbody heating peaks at $\sim 10 \mu \mathrm{m}$ wavelength. As the PL intensity from the Ge QW intersubband transitions is expected to be weak $(<\mathrm{nWs})$, then a modulation technique is required to increase the signal to noise ratio. ${ }^{21}$ The laser illuminates the QWs through an aperture in a parabolic mirror. The sample emission is then collected by this mirror, which couples the emission into the FTIR. The photoluminescence collected is surface normal (x-y). A comparison between the absorption and PL spectra of the $8.1 \mathrm{~nm} \mathrm{QW}$ structure at $6 \mathrm{~K}$ is presented in Fig. 5. It is clear that with the PL the longer wavelength peak is the dominant transition, which might be 


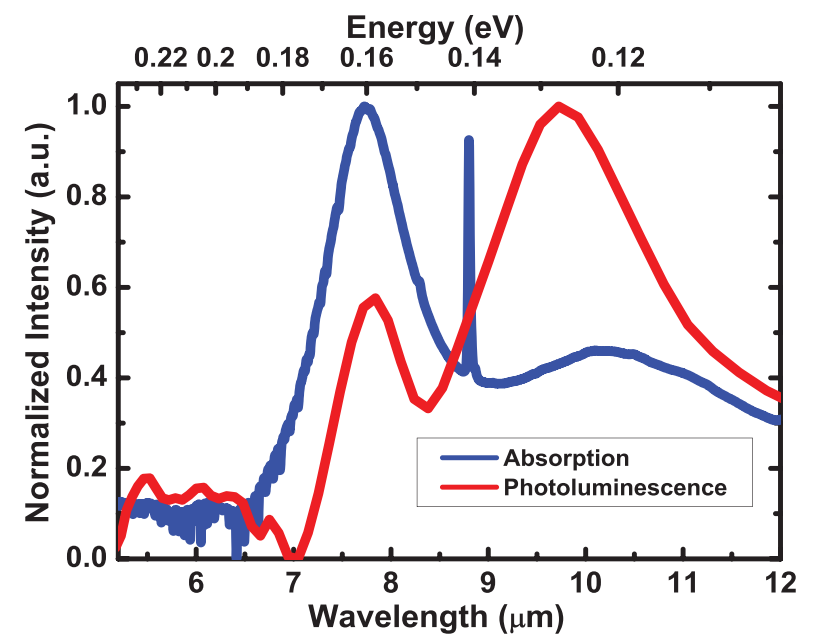

FIG. 5. A comparison between the absorption and photoluminescence spectra for the $8.1 \mathrm{~nm} \mathrm{p}-\mathrm{Ge}$ quantum well structure at $6 \mathrm{~K}$.

arising due to the complex nature of the interband pumping which is used to excite the radiative intersubband transitions. The PL demonstrates a strong temperature dependence, above $30 \mathrm{~K}$, there is no measurable PL for either QW structure due to the increased non-radiative decay rate of the excited states in the valence band.

In conclusion, intersubband absorption from $\mathrm{p}-\mathrm{Ge}$ QWs of different widths designed by an 8-band k.p self-consistent Poisson-Schrödinger tool has been demonstrated. The high quality growth of the Ge QWs has been confirmed through XRD, Raman, and STEM analyses. FTIR transmission spectroscopy displayed absorption peaks corresponding to intersubband transitions between subband states within the QW. The absorption shifts to lower wavelengths for thinner QWs as expected. In addition, the PL spectroscopy agrees well with the FTIR data. The work demonstrates surface-normal intersubband absorption from p-Ge QWs that occurs in the important atmospheric transmission window of $8-13 \mu \mathrm{m}$. It is envisaged that such designs could produce surface normal and waveguide coupled photodetectors for spectroscopic sensing in the MIR.
The research leading to these results has received funding from the European Union's Seventh Framework Programme under Grant Agreement No. 613055 and U.K. EPSRC (Project No. EP/N003225/1).

${ }^{1}$ R. Soref, Proc. SPIE 6898, 689809 (2008).

${ }^{2}$ L. Baldassarre, E. Sakat, J. Frigerio, A. Samarelli, K. Gallacher, E. Calandrini, G. Isella, D. J. Paul, M. Ortolani, and P. Biagioni, Nano Lett. 15, 7225 (2015).

${ }^{3}$ A. Rogalski, J. Appl. Phys. 93, 4355 (2003).

${ }^{4}$ L. Meriggi, M. J. Steer, Y. Ding, I. G. Thayne, C. MacGregor, C. N. Ironside, and M. Sorel, J. Appl. Phys. 117, 063101 (2015).

${ }^{5}$ R. P. G. Karunasiri, J. S. Park, Y. J. Mii, and K. L. Wang, Appl. Phys. Lett. 57, 2585 (1990).

${ }^{6}$ J. S. Park, R. P. G. Karunasiri, and K. L. Wang, Appl. Phys. Lett. 61, 681 (1992).

${ }^{7}$ M. De Seta, G. Capellini, Y. Busby, F. Evangelisti, M. Ortolani, M. Virgilio, G. Grosso, G. Pizzi, A. Nucara, and S. Lupi, Appl. Phys. Lett. 95, 051918 (2009).

${ }^{8}$ D. J. Paul, Laser Photonics Rev. 4, 610 (2010).

${ }^{9}$ G. Isella, D. Chrastina, B. Rössner, T. Hackbarth, H.-J. Herzog, U. König, and H. von Känel, Solid-State Electron. 48, 1317 (2004).

${ }^{10}$ S. Cecchi, E. Gatti, D. Chrastina, J. Frigerio, E. Müller Gubler, D. J. Paul, M. Guzzi, and G. Isella, J. Appl. Phys. 115, 093502 (2014).

${ }^{11}$ P. Boucaud, L. Wu, F. Julien, J.-M. Lourtioz, I. Sagnes, Y. Campidelli, and P.-A. Badox, Appl. Surf. Sci. 102, 342 (1996).

${ }^{12}$ P. Kruck, M. Helm, T. Fromherz, G. Bauer, J. F. Nützel, and G. Abstreiter, Appl. Phys. Lett. 69, 3372 (1996).

${ }^{13}$ D. J. Paul, Phys. Rev. B 77, 155323 (2008).

${ }^{14}$ G. Karunasiri, S. J. Chua, J. S. Park, and K. L. Wang, Mater. Sci. Eng., B 35, 463 (1995).

${ }^{15}$ R. W. Millar, K. Gallacher, A. Samarelli, J. Frigerio, D. Chrastina, G. Isella, T. Dieing, and D. J. Paul, Opt. Express 23, 18193 (2015).

${ }^{16}$ F. Pezzoli, E. Bonera, E. Grilli, M. Guzzi, S. Sanguinetti, D. Chrastina, G. Isella, H. von Känel, E. Wintersberger, J. Stangl, and G. Bauer, Mater. Sci. Semicond. Process. 11, 279 (2008).

${ }^{17}$ P. Kruck, A. Weichselbaum, M. Helm, T. Fromherz, G. Bauer, J. Nützel, and G. Abstreiter, Superlattices Microstruct. 23, 61 (1998).

${ }^{18}$ R. C. Newman, J. Phys.: Condens. Matter 12, R335 (2000).

${ }^{19}$ D. C. Larrabee, G. A. Khodaparast, J. Kono, K. Ueda, Y. Nakajima, M. Nakai, S. Sasa, M. Inoue, K. I. Kolokolov, J. Li, and C. Z. Ning, Appl. Phys. Lett. 83, 3936 (2003).

${ }^{20}$ T. Kotani, M. Arita, K. Hoshino, and Y. Arakawa, Appl. Phys. Lett. 108, 052102 (2016).

${ }^{21}$ S. Sauvage, P. Boucaud, F. H. Julien, O. Gauthier-Lafaye, V. Berger, and J. Nagle, Appl. Phys. Lett. 71, 1183 (1997). 